\title{
A Novel Procedure of Prepuce Reconstruction Customized to the Religious Needs of Some Individuals
}

 \\ ${ }^{1}$ Department of Plastic, Aesthetic and Reconstructive Surgery, and \\ Gender Identity Clinic, Fortis Hospital Shalimar Bagh, Delhi, India \\ ${ }^{2}$ Department of Surgical Oncology and Reconstructive Cancer \\ Surgery, Fortis Hospital Shalimar Bagh, Delhi, India
}

Address for correspondence Richie Gupta, MS, MCh, Department of Plastic, Aesthetic and Reconstructive Surgery, and Gender Identity Clinic, Fortis Hospital Shalimar Bagh, Postal Address A1/11, Jiwan Jyoti Apartments, Kabir Dass Marg, Pitampura, Delhi-110034, India (e-mail: guptarichie@yahoo.com).

\author{
Abstract \\ Keywords \\ - prepuce \\ reconstruction \\ - circumcision \\ - glans coverage \\ - milah \\ - periah
}

Background Authors describe a novel procedure in a group of patients for prepuce reconstruction, ensuring complete glans penis coverage who had either been circumcised in childhood or had congenitally short prepuce.

Methods Case records of all cases done by the novel method which involved penile degloving and maintenance of neoprepuce, with the help of de-epithelization of glans penis and a few key sutures performed over the period from January 2010 to December 2019 were reviewed retrospectively. A total of 46 patients, 32 had congenitally short prepuce and 14 had previous circumcision.

Results All the patients had complete glans penis coverage. None of the patients had complications like urinary infection, meatal stenosis, collection in neoprepucial sac, balanitis, orposthitis. Themeanfollowupwas 23.24 monthsin 37 patients. Ninelosttofollowup. Conclusions The procedure is simple, gives reliable results, and is customized to the needs of the patients. It does not interfere with penile erections.

\section{Introduction}

In modern times, prepuce is not considered a vestigial organ. It is densely innervated and contains erogenous tissue. It is responsible for a part of blood supply to glans penis via frenular artery. The prepuce acts as a roller bearing during sexual intercourse, unfolding, and gliding, thus protecting the glans penis from abrasive and biomechanical stress, as well as helping in retention of lubricating fluids, which facilitates intercourse. ${ }^{1}$ In its absence, the glans becomes less sensitive, rough, dry, and keratinized. Prepuce also has protective role against common microbial infections. Circumcision results not only in the loss of above functions, but it also has psychologic consequences such as a feeling of emotional harm and dissatisfaction, decrease in sexual pleasure, and a sense

DOI https://doi.org/

10.1055/s-0041-1731621

ISSN 0970-0358 of deep mutilation in infancy. ${ }^{2,3}$ Despite this, circumcision is widely practiced in Abrahamic religions such as Judaism and Islam as well as in few Orthodox churches. India has a rich cultural, religious, and social heritage and is the birthplace of many religions. Among these, there are many religious groups who need to shun all material objects including clothing. Any interaction with people, delivery of sermons, travel, etc. must be in cloth-less state. Many of the group members have a congenitally short prepuce, leaving a portion of glans exposed, or have undergone circumcision in childhood in villages for unknown indications. Among these groups, such a condition is considered an ineligibility.

One such religious group approached the authors to device a procedure, which would enable them to achieve complete 
glans penis coverage at all times. Although one of the senior authors has performed prepuce reconstruction for this indication since past 27 years, we have come to the described technique after many refinements. Since past 9 years, we have performed the described procedure with reliable and predictable results.

\section{Materials and Methods}

Case records of 46 cases of young men, with either congenitally short prepuce or childhood circumcision, resulting in exposure of some part of glans penis even in flaccid state, who visited us during the period January 2010 to December 2019 were reviewed for the purpose of this study. Hospital ethical committee had given approval for the study. The patients had the following specific set of instructions for us:

1. They wished to get their glans penis completely covered with prepuce.

2. The glans penis should not be visible even in a state of penile tumescence.

3. There should not be any external scar or stigmata of a surgery.

The patients had come voluntarily for the surgery. Informed consents for surgery were taken from patients, all of whom were in the age group 20 to 45 years. Fourteen of them had been circumcised in childhood in remote villages with no available records. The remaining 32 men had congenitally short prepuce, not fully covering the glans penis, even in a flaccid state. Two patients had coexisting right indirect inguinal hernias.

\section{Technique}

With patient in supine position under spinal anesthesia, pericoronal incision was given. The prepucial remnant, when present, and penile shaft skin were mobilized in the subdartos loose areolar plane to penile base (-Fig. 1). The penile skin dartos flap, well vascularized by superficial and deep external pudendal vessels, could now be advanced well beyond glans tip. Hemostasis was secured. Next, the glans penis skin was de-epithelized from a 5-mm margin all around and parallel to urinary meatus to and including corona ( - Fig. 2). Two

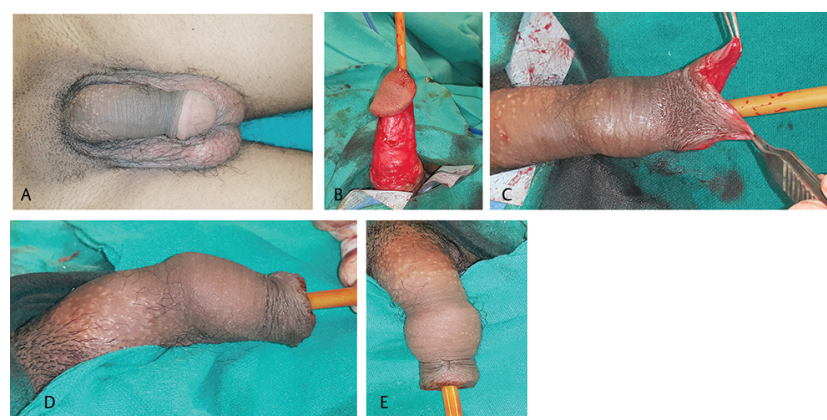

Fig. 1 (A) Patient with congenitally short prepuce and exposed glans penis. Preoperative status. (B) Penile shaft degloved in loose subdartos plane, up to base. (C) Penile shaft skin advanced nearly $5 \mathrm{~cm}$ beyond glans tip. (D) Neoprepuce created with described method, lateral view. (E) Dorsal view. supportive $4 / 0$ vicryl sutures were placed at 12 and 6 o' clock positions to anchor the advanced penile skin flap to coronal ridge and prevent its retraction. As many as 1 to 2 additional sutures were placed if required. The advanced penile skin dartos flap was distally folded inward onto itself for 1.5 to $2 \mathrm{~cm}$, and the folded distal edge sutured to the 5-mm remaining glans epithelial margin around the meatus. The fold in neoprepuce was maintained by two or sometimes three bilayered interrupted 5/0 ethilon sutures. (-Figs. 1, 2). These are also usually placed in 12 and 6 o' clock positions to prevent injury to branches of external pudendal vessels. A gentle compressive dressing is applied. Two patients also had concomitant laparoscopic indirect right inguinal hernia repair. These two patients were primarily catheterized. In both patients, the catheters were removed next day prior to discharge. Follow- up visits were scheduled on days 3, 7 and 14 (-Fig. 3). On day 14, the ethilon sutures were removed. The patients were instructed to apply antiseptic ointment locally for 3 to 4 weeks and keep the neoprepucial sac clean.

\section{Results}

A total of 46 patients underwent the novel procedure. All patients had slight blood-tinged discharge for 2 to 3 days, followed by uneventful healing. No case of hematoma, neoprepuce necrosis, or urinary complications were noted. Erections did occur in postoperative period but did not cause wound dehiscence, penile skin retraction, with loss of neoprepuce or excessive bleeding. After the initial follow-up period, the patients were advised follow-up every 6 months (or whenever they could) for a period of 2 years. Nine patients were lost to follow-up after the early postoperative period ( $<3$ months). The remaining 37 patients had follow-up ranging from 7 months to 10 years and 1 month (mean follow-up 23.24 months) (-Figs. 3 D, 4 A, B). None of
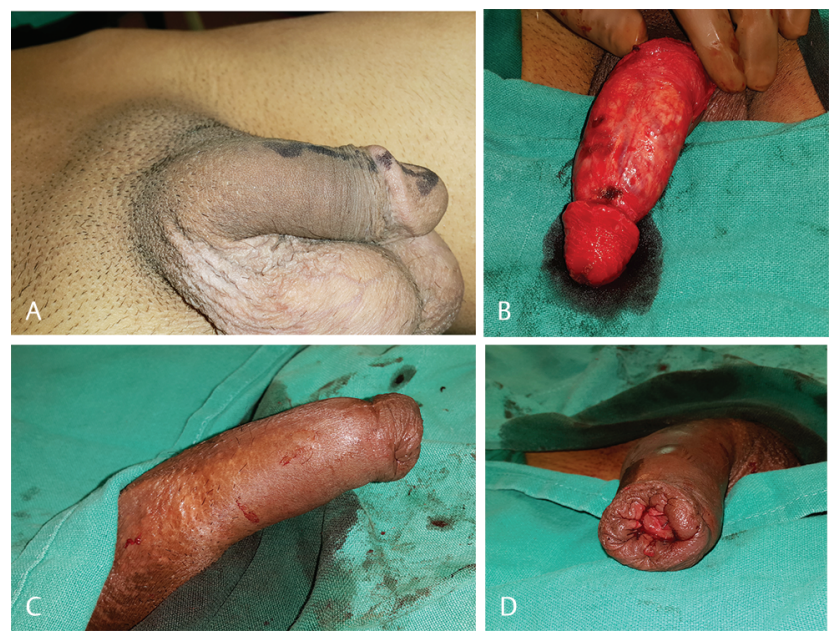

Fig. 2 (A) Another patient with congenitally short prepuce and exposed glans penis. Preoperative right lateral view. (B) Penile shaft degloved up to penile base. Glans penis de-epithelized except a 5-mm margin parallel to urinary meatus. (C) Immediate postoperative right lateral view showing complete glans penis coverage and neoprepuce. (D) End on view showing newly formed epithelial-lined neoprepucial sac. 

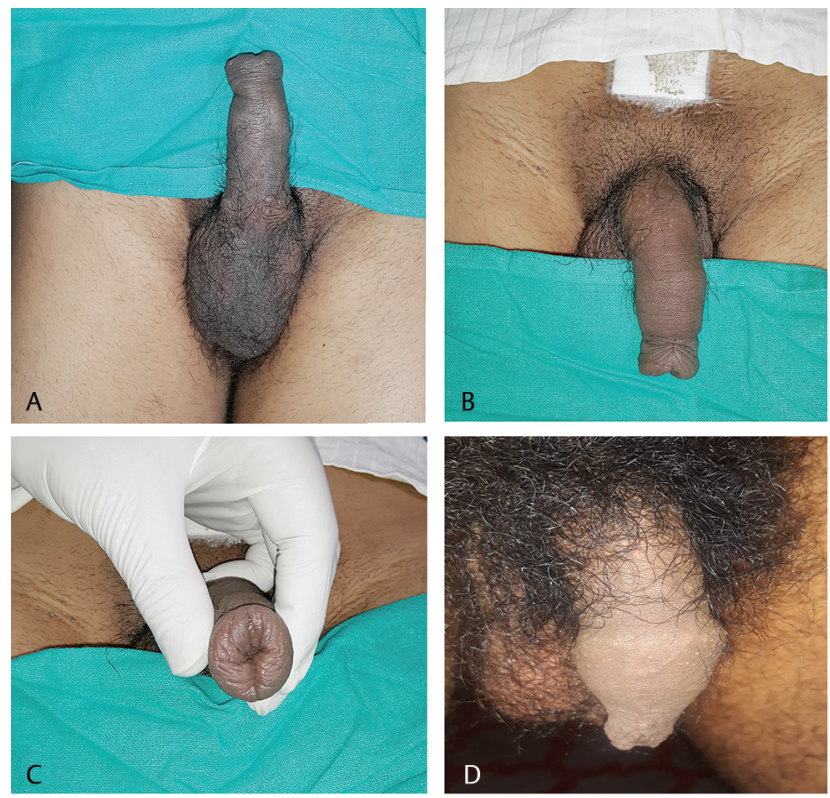

Fig. 3 Same patient as in Fig. 1. One-week postoperative result with some residual edema-(A) Ventral view of penis and neoprepuce. (B) Dorsal view. (C) End-on view showing complete glans penis coverage. (D) One year and 4 months postoperative view.

these patients had loss of definition of neoprepuce. None of the patients had complications like meatal stenosis, urinary infection, balanitis, or posthitis. No patient reported concerns in relation to hygiene of neoprepucial sac. Penile erections and nocturnal/early morning penile tumescence were unaffected by the procedure and painless. Due to the religious propensity of these patients, the sexual activity and penile performance during intercourse could not be evaluated.

\section{Discussion}

Circumcision has been performed since past 6000 years, mainly for religious and medical indications. Based on the identifying feature of a circumcised glans, these individuals have often been subjected to minority stress and oppression. To escape this persecution, some of these individuals underwent the operation of prepuce reconstruction. Celsus was the first to describe two such procedures between CE 14 to 37 in De Medicina (7.25.1). ${ }^{3,4}$ For those with a short prepuce, which was mostly retracted and unable to cover the glans fully, naturally, he suggested the operation of prepucial reconstruction, in which a circumferential incision was given at the root of penis, with extensive forward mobilization of penile shaft skin and distal edge sliding ahead of glans, thus forming an epithelial lined prepuce, which was retained anterior to glans with the help of a tie at distal edge. The denuded penile shaft was dressed till healing by second intention. For those patients who had undergone prior circumcision, he used a pericoronal approach, mobilizing the penile skin superficial to Buck's fascia and stretching it till it covered the complete glans. This formed a prepuce with raw glanular surface, which was dressed till healing by second intention. He called this procedure "decircumcision." Both these procedures left

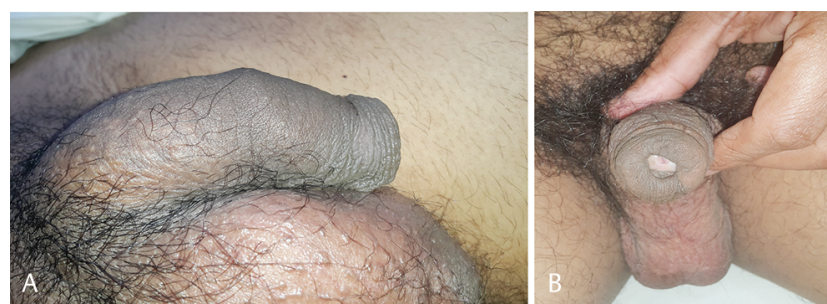

Fig. 4 A 3 years follow-up result-(A) Right lateral view. (B) End-on view showing complete glans coverage and healthy neoprepucial sac.

behind extensive raw areas, and hence there must have been a high incidence of relapse and contracture. There was no significant advancement for next two millennia. Feriz performed prepucial restoration by advancing the penile shaft skin in a manner similar to the Celsus method for patients with short prepuce. ${ }^{3}$ He then buried the raw penile shaft under a tunnel of ventral scrotal skin, released it 10 days later and closed the scrotal defect primarily. Although he operated in Nazi-occupied Holland, he published his technique much later. In 1963, Penn modified the Celsus reconstruction operation for naturally short prepuce by using unmeshed split skin graft to line the bare penile shaft. ${ }^{5}$ Greer's method was similar to that of Feriz. ${ }^{6}$ He covered the bare penile shaft by raising a transverse bridge in midscrotum and burying the penis there, with this bridge covering the bare shaft. At the second stage, a few months later, he raised the penis together with the inset flap covering its shaft. Goodwin modified the Greer procedure by giving three to four small transverse incisions at what would be the new anterior fold of prepuce and closed these longitudinally to narrow it, in an effort to prevent a retraction with loss of the neoprepuce. ${ }^{7}$ He also used $z$ plasties on either side of scrotal skin bridge in the second stage to avoid a straight ventral penile suture line. Besides being two-staged, this procedure transfers thicker and hair-bearing scrotal skin onto penile shaft, which is unesthetic. To reduce the morbidity of two stages, Lynch used a longitudinal midline flap from scrotum, but other disadvantages of a scrotal flap remained. ${ }^{8}$ Brandes and McAninch suggested that the McAninch method of circular penile fasciocutaneous flap for reconstructing extensive urethral strictures could be modified, and instead of ventrally dividing the skin island, the circular skin island be transposed distally and folded inward. ${ }^{9}$ The raw outer aspect was skin grafted to form a prepuce. Alternatively, they suggested developing a circular Buck's fascia flap without this skin island, pulling it distal to glans by several centimeters and folding it inward on itself. Both the inner and outer surfaces of this flap are then skin grafted. It is not clear however from their paper, if actual cases were done, or they suggested this as just a concept. The paper does not have any pre and postoperative photographs or patient data. Conceptually, this method incorporates biplane mobilization-a superficial dartos plane and a plane deep to Buck's fascia with extensive separation and mobilization of superficial Buck's fascia from neurovascular bundles. While this method has been applied in urethral strictures, it is inconceivable that the tough Buck's fascia would be able 
to stretch distally to the extent required in a case of neoprepuce reconstruction. Also, given the extent of mobilization required, there would be a high probability of neurovascular injury and hematoma. The vascularity of neoprepuce would be unpredictable, and chances of penile shaft skin necrosis if superficial plane of dissection is too superficial. If such a procedure were to succeed, the patient would still have a skin grafted neoprepuce, a stigma of such a surgery having been performed, and thus unsuitable for our group of patients.

Efforts are on with tissue-engineering techniques to develop a prepuce. Purpura developed a decellularized extracellular matrix-based biomaterial scaffold from human foreskin samples. ${ }^{1}$ But this will need to be seeded with host cells and then overcome by the holy grail of tissue engineering-a functional microvascular system, to enable it to be transplanted. Even preclinical animal model work is yet to be performed. There is a plethora of modern day "Pondum Judeus" type of devices developed for tissue expansion of residual prepucial and penile shaft skin. These devices must be worn for 14 to 16 hours a day for months or years altogether, with unpredictable results. Often, once the devices are removed, the expanded skin retracts. These have been successful in only a handful of cases.

Our patients have a specific set of requirements for religious reasons. Any exposure of glans penis due to a short prepuce or prior circumcision (even in a state of penile tumescence) is not acceptable to them. Also, any stigma of genital surgery in the form of a scar, skin graft, or flap on the penile shaft would be a giveaway that such a surgical procedure has been performed. Authors are familiar with the elastic properties and vascularity of penile skin dartos flap, having used this on hundreds of occasions in cases of neovaginoplasties in transwomen, either alone, or together with perineoscrotal flap. ${ }^{10}$ Prior to January 2010, authors performed advancement of penile skin dartos flap together with placement of bilayer sutures to maintain the fold and sutured the folded distal edge to pericoronal area. However, the results were unpredictable. The neoprepuce would retract to variable extent and often part of glans penis would subsequently be exposed. The authors were already familiar with the procedure of de-epithelization of clitoral glans,$^{10}$ which they had performed in numerous female-to-male transsexuals. They extended this procedure to penile glans in the current group of patients for providing a large area of adhesion between advanced penile-skin dartos flap and the de-epithelized glans penis, which would serve to maintain the advancement and result in a nonretractile neoprepuce, ensuring complete glans penis coverage at all times.

\section{Conclusion}

Author's procedure for prepuce reconstruction for religious need is simple, gives reliable results, and is customized to the needs of their patients. The procedure does not interfere with penile erections, but penetrating sexual performance was not evaluated. The procedure may be useful in reconstructions following circumcision, neoplastic resection and trauma.

\section{Conflicts of Interest}

None declared.

\section{References}

1 Purpura V, Bondioli E, Cunningham EJ, et al. The development of a decellularized extracellular matrix-based biomaterial scaffold derived from human foreskin for the purpose of foreskin reconstruction in circumcised males. J Tissue Eng 2018;9:2041731418812613

2 Hammond T, Long term consequences of neonatal circumcision: A preliminary poll of circumcised males. In: Sexual Mutilations: A Human Tragedy. New York: Plenum Press,; 1997

3 Schultheiss D, Truss MC, Stief CG, Jonas U. Uncircumcision: a historical review of preputial restoration. Plast Reconstr Surg 1998;101(7):1990-1998

4 Rubin JP. Celsus's decircumcision operation. Urology 1980;XVI(1):121-124

5 Penn J. Penile reform. Br J Plast Surg 1963;16:287-288

6 Greer DM Jr, Mohl PC, Sheley KA. A technique for foreskin reconstruction and some preliminary results. J Sex Res 1982;18(4):324-330

7 Goodwin WE. Uncircumcision: a technique for plastic reconstruction of a prepuce after circumcision. J Urol 1990;144(5):1203-1205

8 Lynch MJ, Pryor JP. Uncircumcision: a one-stage procedure. $\mathrm{Br}$ J Urol 1993;72(2):257-258

9 Brandes SB, McAninch JW. Surgical methods of restoring the prepuce: a critical review. BJU Int 1999;83(Suppl 1):109-113

10 Gupta R, Gupta R, Mehta R, Aggarwal A, Mathur A, Gender dysphoria. In K Agrawal and R K Mahajan, eds. Textbook of Plastic, Reconstructive and Aesthetic Surgery Volume IV (259-293). Noida, Uttar Pradesh: Thieme Publishers; 2019 